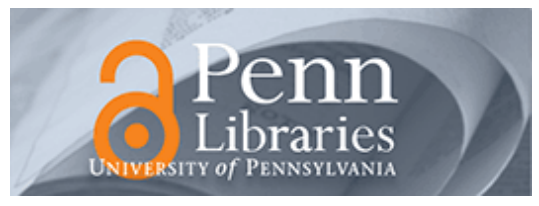

University of Pennsylvania

ScholarlyCommons

Management Papers

Wharton Faculty Research

2012

\title{
Healthcare Innovation Across Sectors: Convergences and Divergences
}

Lawton R. Burns

University of Pennsylvania

Stephen M. Sammut

Follow this and additional works at: https://repository.upenn.edu/mgmt_papers

Part of the Management Sciences and Quantitative Methods Commons

\section{Recommended Citation}

Burns, L. R., \& Sammut, S. M. (2012). Healthcare Innovation Across Sectors: Convergences and Divergences. The Business of Healthcare Innovation, 348-364. Retrieved from https://repository.upenn.edu/mgmt_papers/241

This paper is posted at ScholarlyCommons. https://repository.upenn.edu/mgmt_papers/241

For more information, please contact repository@pobox.upenn.edu. 


\title{
Healthcare Innovation Across Sectors: Convergences and Divergences
}

\author{
Abstract \\ All of the sectors analyzed in this volume face the same dual challenge: the invention of new technology \\ and assuring its long-term clinical adoption by customers. These challenges are neither easy nor \\ inexpensive. For many of the sectors, the technology and the underlying science have encountered the \\ same phenomenon as other technology development in other endeavors, namely convergence of many \\ skills. Pharmaceutical and biotechnology firms - long accustomed to both random discovery and \\ synthesis of bioactive chemicals or recombination of known active proteins - are now relying on genomic \\ and proteomic foundations for drug discovery. These new sciences are just the first steps in the long \\ process of drug development wherein tools such as bioinformatics must be integrated. As companies in \\ the sector pursue new avenues of discovery and development, and as the associated costs spiral ever \\ upward, healthcare systems throughout the world seek to rationalize care and lower overall costs. The \\ industry has the added burden, therefore, of demonstrating the economic advantages of new drugs, thus \\ giving rise to yet another new discipline, pharmaco-economics. \\ Disciplines \\ Management Sciences and Quantitative Methods
}




\section{Healthcare innovation across sectors: convergences and divergences}

\section{Lawton R. Burns and Stephen M. Sammut}

The twin towers: invention and adoption

Common business models

Strategic resources, capabilities, and key success factors

Technological convergence across sectors

\section{The twin towers: invention and adoption}

All of the sectors analyzed in this volume face the same dual challenge: the invention of new technology and assuring its long-term clinical adoption by customers. These challenges are neither easy nor inexpensive.

For many of the sectors, the technology and the underlying science have encountered the same phenomenon as other technology development in other endeavors, namely convergence of many skills. Pharmaceutical and biotechnology firms - long accustomed to both random discovery and synthesis of bioactive chemicals or recombination of known active proteins - are now relying on genomic and proteomic foundations for drug discovery. These new sciences are just the first steps in the long process of drug development wherein tools such as bioinformatics must be integrated. As companies in the sector pursue new avenues of discovery and development, and as the associated costs spiral ever upward, healthcare systems throughout the world seek to rationalize care and lower overall costs. The industry has the added burden, therefore, of demonstrating the economic advantages of new drugs, thus giving rise to yet another new discipline, pharmaco-economics.

At the same time, the sectors must increasingly conduct their R\&D activities with an understanding of multiple technologies. Pharmaceutical and biotechnology firms must embrace not only genomics and proteomics, but also the more traditional technologies that are chemistry-based. Platform-based firms 
are constrained to advance beyond their circumscribed technological base to incorporate development and delivery capabilities. Device firms have migrated beyond electronics to encompass various materials sciences and information systems, and information technology (IT) firms have begun to combine their products with imaging and broadband capabilities.

The increasingly complex milieu of discovery and development has the added challenge of satisfying the needs of an aging and more health conscious community of patients. Physicians, therefore, must remain current with new technology as never before and must determine how the new therapies can be incorporated into their practice in such a way that payers exerting increasing levels of control will approve.

To a great extent, the firms are built on the intangibles of their intellectual capital and the ability to harness and coordinate it across different therapeutic areas and research programs. Not surprisingly, several of the sectors rest heavily on the art of discovery and the vagaries of trial-and-error experimentation.

Intellectual property law in the developed nations has always been the foundation of pharmaceutical economics, wherein companies could depend on a limited monopoly for their patented synthetic compounds. There are many factors conspiring to the resulting hegemony in each therapeutic area for both traditional pharmaceutical companies as well as biotechnology companies. These factors merit review in this summary chapter, because the fundamental business strategies will be affected by a sea change in intellectual property regimes and necessary new approaches to managing intangible property.

Historically, the discovery and development of a new compound was the effort of each company. A company had sole ownership of a chain of patent blockades for each compound from lab bench to the scaled-up synthesis for production. The drug discovery and development intellectual property inventory has grown increasingly fragmented, however. The proliferation of competent university technology transfer programs and the global emergence of research-driven biotechnology companies - now in the thousands in the US, Europe, India, China and Taiwan, Singapore, South Africa, Brazil, and former Warsaw Pact countries - has produced a patent landscape that requires a dozen or more technology licenses for each product brought to market, with a resulting layering of royalty obligations and consequent reduction of profit margins. These factors are compounded by the concerted efforts of developing countries to secure products at affordable costs for their health-stressed populations. The pharmaceutical companies acquiesce with deeply discounted or donated products, only to find that black markets in those countries emerge and export the same products at below market prices 
to the developing world, thus undermining attempts at health equity. And the industry is cast as avaricious and must, therefore, address issues that now extend from the challenges of drug development through commerce to unprecedented issues of ethics and morality. There is a cruel irony here; the industry's contribution to human health over the last half-century is inestimable and the professionals in the industry pride themselves on their commitment to doing good while doing well. The controversy, however, will not be soon resolved.

The invention of new technology and the securing of proprietary rights to assure a return on investment are only half of the equation. The other half is its successful commercialization and adoption by customers and buyers ideally as a new standard of care. The challenge here varies, depending on the sector. Some sectors have succeeded largely due to their commercialization efforts, such as the pharmaceutical sector's development of large sales forces and sophisticated marketing techniques. But pharmaceuticals' success here has been financed by two decades of strong earnings that are not enjoyed by other sectors such as the still emerging biotechnology sector, where scarcely two dozen companies of thousands globally have achieved profitability. In contrast to pharmaceuticals, biotechnology firms have spent more of their revenues on $\mathrm{R} \& \mathrm{D}$ activities as compared to 15 percent to 20 percent spent by the pharmaceutical sector (although in absolute numbers the R\&D spending of the largest pharmaceutical companies rivals the cumulative spending of the greater than 300 US publicly traded biotechnology companies).

Given the cumulative losses referred to in chapter 3 and the mere handful of successful products, biotechnology companies have not enjoyed the financial slack to invest in both R\&D and the infrastructure for commercialization, such as detailing forces and advertising. This limitation has largely condemned the majority of biotechnology companies to retreat from being fully integrated pharmaceutical companies (FIPCOs) to the model of research-intensive pharmaceutical companies (RIPCOs). While it is the case that biotechnology firms from their origins worked with pharmaceutical firms, for example, Genentech's collaboration with Eli Lilly for the development of recombinant human insulin, the pace and absolute number of joint commercialization efforts with pharmaceutical firms has moved markedly upwards.

The issues above have a curious metric expressed in the capital markets. Burrill \& Company has tracked the market capitalizations of the entire group of public biotechnology companies against the combined market capitalization of Merck \& Company and Pfizer. Over the decade the ratio of market capitalizations has been in the range of 0.7 to 1.1 . In other words, the public 
markets value the commercial infrastructure and FIPCO model of established pharmaceutical companies (despite their challenged product pipelines) far more than the research pipelines (over 350 products in clinical trials) of the biotechnology industry as a whole.

As described in chapter 4, platform technology and IT firms face the issue of strained resources and severely decreased market capitalization, largely because the bulk of firms in these sectors are smaller, entrepreneurial startups that have focused heavily on new genomic and proteomic approaches to drug discovery only to find that the required array of technologies and skills is so fragmented that their point in the value chain cannot extract sufficient rents from the pricing of pharmaceuticals to sustain their business models.

Across the five sectors characterized in this book, medical device firms are best positioned to deal with the two challenges of innovation and commercialization. Companies such as Medtronic, Guidant, and several operating companies of Johnson \& Johnson have a documented stream of innovative products - often revolutionary in terms of less-invasive life-saving intervention - and a strong history of earnings to finance product development and commercialization activities. Device companies are not immune from the changing nature of intellectual property regimes, but their use of and reliance on patents differs from the pharmaceutical industry. Each product line is often covered by scores of patents often controlled by each company - for example, the patent blockades assembled by each of the above cited participants in the balloon angioplasty and vascular stent business.

Until the early 1990s pharmaceutical firms enjoyed a growth rate in earnings the pace of which began to decline with the downturn in drug productivity. Like pharmaceutical firms, device companies market their products directly to physicians. Unlike pharmaceutical firms, device firms enjoy a shorter and less costly regulatory path and far more efficient marketing channels by virtue of dealing with a small number of specialists with high volumes in a given therapeutic area (as opposed to marketing to a large number of primary care physicians). There are also other differences in the marketing dynamics. The most successful devices over the last two decades have created new procedures that carry fees for physicians and hospitals that drive sustained adoption. Patients have clearly been the beneficiary of the new technologies, as well as the national health care bill, as the number of open heart and renal calculi surgeries have declined dramatically with the introduction of less-invasive procedures.

Less tangible is the relationship between device company detailers and their physician customers. Here there is a far greater two-way dialog whereby 
companies are fed with a continuous stream of new ideas or ways of improving their products. While there are advantages to participation in the medical device sector, it is also the most competitive and litigious sector. Entry into the market is enormously difficult, but when successful, the younger companies are rewarded with extraordinary acquisition deals. For example, Medtronic acquired Minimed, with modest sales for over $\$ 1$ billion. Why? Minimed had technology for blood glucose monitoring that was an important key to Medtronic's product strategy in diabetes. In similar manner, Johnson \& Johnson paid \$14 billion for ALZA, which had only $\$ 1$ billion in sales. Why? ALZA's drug delivery and controlled-release technologies, which had been utilized largely through a network of alliances with pharmaceutical companies, would provide J\&J's divisions with dramatic proprietary means of delivering their own drugs. To close the circle, J\&J incorporated ALZA technology into the coating of vascular stents with antirestenosis factors.

In contrast to the more definable commercial environment and fully integrated business models of the device sector, both platform technology and bioinformatics firms seem less well positioned to deal with these dual challenges. Each sector faces difficulties in developing new technology that is dependent on integration with other technologies (including change management in the case of biotechnology and healthcare information systems) to be useful. This need to integrate among companies conspires against a privileged and patent-protected position in the value chain; the demonstration of problem-solving capabilities to end users is lost in a morass of complexity and competition.

Biotechnology firms pursuing drug discovery and development seem to occupy an intermediate position on the spectrum. While in the past they certainly experienced challenges with both innovation and adoption, they are emerging as the solution to the productivity problems faced by pharmaceutical firms in terms of developing new, innovative products. As chapters 2 and 3 noted, the majority of the promising drug candidates of the future are being sourced from the biotechnology sector. Moreover, given the novelty of the new therapeutics emerging from the companies addressing hitherto untreatable debilitating diseases that affect smaller patient populations, such as rheumatoid arthritis (monthly costs for the new drugs exceed $\$ 1000$ ), and given the current ambiguity of market entry of "biogenerics," these firms have thus far faced much lower pricing resistance from payers than do the makers of synthetic pharmaceuticals that have focused on chronic diseases that affect large portions of the population. Nevertheless, a major challenge going forward for the biotechnology companies will be the integration of genomic-based technologies into the practices of physicians. 


\section{Common business models}

Another convergence evident across sectors has been the pursuit of common business models. Many of the sectors have undergone rapid transitions in their business models toward fully integrated companies. Biotechnology and platform technology firms are both migrating away from a strict focus on research toward the inclusion of drug development and commercialization activities. In this manner, they are striving to achieve the FIPCO model already prominent in the pharmaceutical sector and among the large medical device firms. One obvious driver of this trend is the need to confront the dual challenges of innovation and adoption discussed above and ultimately to enjoy higher price-earnings multiples and thus a lower cost of equity capital. Another common business model has been growth via mergers and acquisitions (M\&As). Across the sectors, firms have used M\&A in an effort to leapfrog the competition, facilitate convergence of complementary technologies, increase their attractiveness as a strategic partner (e.g., for licensing in pharmaceuticals), diversify into new therapeutic areas (e.g., pharmaceutical firms, device firms) or new complementary technologies (e.g., IT firms), or to achieve scale economies (whether real or imagined). Several of the sectors adhere to a belief in the value of large scale in their operations. Evidence from chapter 5 questions the presumed benefits, at least among pharmaceutical firms. There is unpublished evidence that M\&As among medical device firms also do not translate into abnormal stock returns. ${ }^{1}$

The M\&A model has also been utilized to sustain growth rates in increasingly large firms that have found it difficult to grow organically. This is particularly true for those sectors with proportionately larger amounts of public equity and thus great pressures for quarterly earnings. M\&A satisfies the demand for earnings growth by pooling the earnings of the merging firms and rationalizing $\mathrm{R} \& \mathrm{D}$ programs, general and administrative costs, and detailing costs, thus forestalling or dampening the need for internal, organic growth.

The M\&A model is also another common strategy used in dealing with a fragmented market structure. A merger has the effect of reducing the number of competitors by one. As Porter's "five forces" analysis (see chapter 1) suggests, mergers reduce market rivalry and potentially lessen price competition, thereby increasing the ability of incumbent firms to earn above-average profits.

The chapters in this volume suggest that most sectors have fragmented market structures, that is, lots of competitors with small market shares. 
This is clearly true for biotechnology firms, platform technology firms, and IT firms. The pharmaceutical sector has undergone a decade of consolidation and become more concentrated than before; however, no single firm enjoys greater than 10-11 percent market share. The medical device sector is the most consolidated of all, with three very big players in what the author of chapter 6 describes as an oligopoly. Nevertheless, all five sectors are quite innovative, lending further confirmation to the observation in chapter 5 that the market structure of an industry does not seem strongly correlated with the innovativeness of the firms within it. It is possible, of course, that all of this M\&A activity might diminish innovation by virtue of erecting entry barriers to the industry and/or by consuming the attentions and energies of incumbent firms. In keeping with the Porter paradigm, fewer major players means fewer companies with which to partner biotechnology companies, thus tilting the economics of alliances in favor of the larger incumbents.

In addition to the fragmented sectors, most of the market sectors examined here are modest in size relative to pharmaceuticals, but are expected to grow significantly over the next few years. Worldwide, the sales of pharmaceuticals were pegged at nearly $\$ 500$ billion in 2003 , compared to $\$ 56$ billion for biotechnology products and $\$ 75$ billion for devices, and were estimated at $\$ 100$ billion for IT in 2005.

\section{Strategic resources, capabilities, and key success factors}

The strategic management literature (in particular, the resource-based view of the firm) places a heavy emphasis on strategic capabilities as keys to competitive advantage. These capabilities are based on combinations of "resources" and "routines" that are unique to a firm. Resources can be both tangible (capital, balance sheet strength, physical plant, and equipment) and intangible (intellectual capital, reputation, innovation potential, employee motivation, culture). The routines are processes for coordinating the resources in productive ways (e.g., harmonizing social and technical systems, teamwork, and other integrative mechanisms) that other firms find difficult to do or emulate.

\section{Resources}

What are the strategic resources in the sectors examined here? Based on the prominence of risk, capital, and long cycle times in many of these sectors, the 
amount of financial resources is clearly important. Firms that generate high earnings, such as pharmaceutical and device companies, can rely on their own internal cash flows to finance $\mathrm{R} \& \mathrm{D}$, rather than be subject to the vagaries of private equity and the IPO marketplace. In addition to being more predictable, cash flows provide lower cost capital. Such cash flows have been found to be associated with higher levels of R\&D investment in the pharmaceutical sector. In the other sectors, by contrast, firms tend to be smaller start-ups in continuing need of capital to grow.

Scale is another, related resource of importance. Scale enables a firm to develop geographic scope in its marketing and commercial activities: for example, sell its products more broadly, target more customers and do so more intensively. Scale also improves the attractiveness of a firm as an alliance partner, and thereby provides an advantage over other firms in accessing new technologies and products from smaller firms.

Established and efficient sales channels might also be considered an important resource for competitive advantage, partially by serving as a barrier to entry to smaller firms. Many of the products and technologies discussed in this volume are marketed to physicians and other providers. Like the sectors discussed here, these buyer markets are fragmented and not centrally accessible. Large sales forces with detailed understanding of the clinicians being targeted and historical relationships of support are typically required for success.

There is, finally, a subtle concept in the resources literature, and that is the notion of fungibility of resources, as distinct from the concept of "ambidextrous" companies described below. The term basically means the ability of a firm to apply a resource or capability in one area of its business to another, thereby accelerating development of new business activity or achieving greater production economies across a firm. The mechanics of fungibility vary greatly from one sector to another, but biotechnology has a version of fungibility that a few companies in the sector discovered early in the history of the industry. For example, the aforesaid relationship between Genentech and Eli Lilly meant that Genentech would abandon the insulin market to Eli Lilly, the dominant provider of the soon-to-be obsolete porcine insulin but also the controller of relationships with internists and diabetologists. The arrangement, however, had the obvious benefit of providing Genentech with critically needed cash. The less obvious benefit was that Lilly effectively financed the development of Genentech's know-how for production and scale-up (major issues for biopharmaceuticals in the 1980s) that could be used across its pipeline of products. Lilly's knowledge of regulatory matters and its credibility with the Food and Drug Administration also promoted the creation of standards for the 
evaluation of recombinant products, with Genentech and Amgen (to say nothing of the public) being the major beneficiaries.

\section{Organizational routines and capabilities}

Beyond these resources, there are a series of organizational routines and capabilities that seem critical for success. One important routine is the ability to manage knowledge across a firm's silos (the distinct departments or organizational enclaves for portions of the discovery, development, or marketing processes) and projects, both within and across therapeutic areas. The leveraging of knowledge and the insights thereby gleaned produce one set of the synergies expected from diversification efforts. This is no easy task, as professional firms in other knowledge-intensive industries (e.g., medicine, academia, consulting) have discovered.

Firms in the pharmaceutical, device, and IT sectors nevertheless appear to rely on these presumed advantages as one justification for their diversification activities. As chapter 5 describes, the activity requires a host of integrative mechanisms to bring together individual expertise, departmental silos, scientific disciplines, development projects, and stages in the internal value chain, namely, research, development, manufacturing, marketing. A cardinal principle of management theory has long held that the degree of internal differentiation within a firm must be matched by the requisite amount of integration across laboratories, operating units, and divisions - another spin on the concept of fungibility. Diversification thus necessitates integration.

Executives and managers in the firms profiled in this volume face some daunting prospects here. First, diversification is often pursued via M\&A strategies. The qualitative evidence on M\&As suggests that top executives until recently - place heavier emphasis on the merger transaction than they do on postmerger integration. Failure to attend to the latter will diminish the prospects for achieving any synergies, assuming that the synergies were honestly assessed during merger planning.

Second, integration is time-consuming, meeting-intensive, and difficult work. It also affects the power equation among executives and requires the emotionally wrenching problem of reducing staff and closing plants. While it may be a cynical observation, senior executives often address the impact of mergers on the issues of shared power among the same senior executives following the actual merger. Executives at lower levels are often left to fend for themselves. These are among the reasons why top executives often delegate integration to lower level executives or engage outside consultants 
to develop - and sometimes implement - rationalization plans. Outside consultants are often used to study and rationalize $R \& D$ projects and product lines in order to comply with the requirements of antitrust authorities. Evidence from the strategy literature suggests, however, that integration activities and efforts to retain the intellectual capital from the firm acquired are the two most important predictors of M\&A success.

Third, the literature on corporate diversification is mixed, at best. Diversification, of course, is definable only on a case specific basis. On the one hand, companies can diversify, essentially augment, a product line by adding new drugs that fit into a detailing call pattern to the same physicians. On the other hand, diversification can take the form of adding entirely new lines of business, albeit in the same industry, justified on the basis of a portfolio approach to risk mitigation. The pharmaceutical sector has had a curious history of this latter type of diversification. During the 1970s many of pharmaceutical companies redefined themselves as broad human care companies and diversified into diagnostics systems and services, medical devices, hospital supplies, laboratory instrumentation, dental and optomology products, over-the-counter pharmaceuticals, nutritional supplements, and even cosmetics. It is hard to establish technological synergy between surgical instruments and lipstick. This human care conglomeration did not work, and the companies systematically began divesting all nonpharmaceutical businesses. Perhaps the only company in the industry to achieve successful diversification is Johnson \& Johnson. The basis of their success is a topic for another large book, but suffice it to say, their operational and marketing insight is managed across the corporation, and the company is disciplined to know when it can win and when it cannot. Why does J\&J sell toothbrushes but not toothpaste?

Diversification is often pursued and justified, therefore, for reasons of scope economies. The underlying assumption, however, is that each functional unit is sufficiently linked to other units in ways that they can equitably share the economies. Integration of new systems often interrupts the status quo of transfer pricing among departments and the related margins for those departments. Again, not to be cynical, but managerial compensation and promotion are determined by each unit's performance. Units and divisions within corporations compete, and the pharmaceutical sector is not an exception to this dynamic. In fact, the managerial structure of the pharmaceutical sector differs from other industries. True profit and loss responsibility exists at only the highest levels of a corporation. At lower levels there is virtual profit and loss responsibility for the product and brand managers. The other 
operational silos, such as discovery, development, and manufacturing, are cost centers. In cases where scale economies are effectively a zero-sum game, senior management cannot assume cooperation among divisions unless the compensation standards are revised. This may explain why firms that pursue "related diversification" do not necessarily perform better than those that pursue "unrelated diversification." Instead, the literature suggests that some modicum of diversification is correlated with firm performance, but not with excessively high or low levels. ${ }^{2}$

Another key capability is portfolio management and optimization, as alluded to in the discussion of M\&A. This is clearly a major issue facing the pharmaceutical sector today: specifically in which new products should a company invest, and which existing products should be milked, further developed, or divested. The participants in the medical device sector confront these issues differently from the pharmaceutical sector; their decision points are sharper by virtue of more rapid changes in marketing performance and technological substitution. The device sector will confront these issues more frequently, given the number of emerging and unexplored clinical areas outlined in chapter 6 .

A key capability in portfolio management and optimization is factoring two sources of uncertainty: market uncertainty (is there a market?) and technical uncertainty (can the firm deliver?). These two types of uncertainty parallel the two key challenges discussed at the beginning of this chapter. As a solution to this dual management problem, strategy theorists as well as industry practitioners have relied on real options reasoning rather than on net present value calculations. In the real options approach, firms distinguish among the available technological opportunities available to them, manage them differently, and then learn from them for purposes of the next round of investments. The real options framework has the additional benefit in this industry of forcing the formulation of process milestones that have the effect of establishing decision criteria and the mitigation of financial risk by portioning the development process into predefined stages.

For example, Ian MacMillan of the Wharton School has devoted considerable effort to studying how firms decide among technological opportunities which ones to fund and staff. ${ }^{3}$ In an analysis of a medical device firm, he first identified the current portfolio of investment projects along the two types of uncertainty. He found that the grid was overcrowded with more projects than the firm could staff or finance, and overly invested in highly demanding, new platform launches. Such a situation is typically found in other firms and industries. To correct the problem, he has developed a grid of five strategic 
options for firms to consider based on these two types of uncertainty: enhancement launches (add new attributes to existing platform - low on both types of uncertainty), new platform launches (medium uncertainty), positioning options (can fail on the dimension of technical uncertainty), scouting options (can fail on the dimension of market uncertainty), and stepping-stones (can fail on both technical and market dimensions). The problem then becomes one of strategic allocation of finite resources across the five options. The solution MacMillan develops is to identify a separate resource pool for each type of option, recognize that each type has its own timing pattern, and then allow competition for resources among opportunities within (but not across) option groups. In concert with executives in the semiconductor industry, MacMillan argues that strategic allocation of resources is the key task of entrepreneurship. ${ }^{4}$

In a similar vein, some pharmaceutical firms are refocusing their $R \& D$ efforts on a smaller number of projects and therapeutic areas. In effect, they are dediversifying - and thereby recognizing some of the problems of diversified activity noted above. In combination with this more focused approach, they are also developing multidisciplinary and multifunctional silo teams on a global basis to coordinate product development, along with smaller groupings of people. In effect, they are also recognizing the importance of integrative mechanisms and the value of small scale (e.g., as found in the biotechnology sector) for innovation.

Another key capability is the management of strategic alliances and collaborations along the value chain. The chapters in this volume suggest that success in the pharmaceutical, biotechnology, and platform technology sectors all hinge on alliance formation and performance. Perhaps this observation should sound the alarm. As numerous management scholars have pointed out, alliance formation and performance is a behavioral science skill - and as such is more art than science. Indeed, one scholar has likened it to dating. ${ }^{5}$ It should thus not be surprising that the success rate with strategic alliances in industry (roughly a 50 percent "instability rate," defined as an unplanned and premature change in alliance relationship status) parallels the success rate of marriage. And even marriages that endure are not necessarily happy ones!

The strategic literature informs us as to the critical ingredients for a successful strategic alliance (echoing some of the ingredients mentioned in chapters 2 and 3). To reiterate one of the themes above, alliances are knowledge-creating networks. Their success depends heavily on processes of knowledge creation and management, organizational sharing and learning, conflict resolution, and trust building. ${ }^{6}$ Perhaps even more importantly, like the M\&As discussed in 
chapter 5, alliance success hinges on due diligence in selecting the proper partner up front.

The management of collaborations is also implicit in efforts to make M\&As work. There is a growing body of qualitative evidence on the postmerger processes that need to be in place, particularly those that serve to retain the human resources talent in the acquired firm and to merge the different cultures of the merger partners. Both factors have been identified as key contributors to M\&A success or failure. ${ }^{7}$

Another important capability mentioned in chapter 1 and reiterated in several forms throughout the volume is managing the balancing act. In addition to managing the dual challenges noted at the outset, smaller start-up firms (e.g., in the biotechnology, platform technology, devices, and IT sectors) must balance their $\mathrm{R} \& \mathrm{D}$ investments with maintaining sales momentum to thus avoid becoming the target of acquisition by a larger competitor.

At a more conceptual level, the innovation literature has suggested the need to balance a firm's short-term focus on earnings and operating efficiency with a long-term focus on research, discovery, and experimentation. Innovation scholars argue that "ambidextrous" firms - firms that can simultaneously pursue these two, contrasting orientations of "exploitation" and "exploration" - are more likely to succeed. ${ }^{8}$ The ambidextrous approach requires two different types of change processes (short-term adjustments versus long-term adaptations), goals of change (maximize economic value versus develop firm capabilities), methods of planning (programmatic versus emergent), targets of change (structure/systems versus culture), directions of change (top-down versus bottom-up), methods of change (imitation versus experimentation), and scales of change (small scale versus large scale).

This balancing act will prove difficult for most firms. The ambidextrous approach requires two different mindsets not likely found in the same executive. Thus, firms need an executive team with both mindsets in some balance. Moreover, most firms may not have (or do not perceive they have) the luxury to pursue the exploration side, given Wall Street pressures for short-term earnings growth. Exploration may also be inhibited by CEO compensation packages and incentives from the Board, the tendency to outsource strategic planning to consultants, the short tenures of CEOs, the tendency of CEOs to subscribe to programmatic change methods, and the associated tendency to deemphasize local level experimentation in large firms. ${ }^{9}$

Perhaps the most important capability that will be required in the future is "affordable innovation" as described in chapter 2. Following the value chain perspective, producers across all sectors may confront a payer community 
that is increasingly activist in documenting the value (price for quality) of the products they pay for. There are already signs of this in Europe for both pharmaceutical and device products. There have been rumblings of this developing in the US, although a recent attempt to tie head-to-head clinical performance of drugs to Medicare reimbursement was scuttled (revised approach may emerge, however).

The pressures here for affordability and performance are keenly felt in the IT sector, where the costs of replacing legacy systems are enormous. They are also growing in the pharmaceutical sector, given the need to monitor drug reimportation and track and recalibrate reference pricing on a real-time basis. Pressures for more affordable products have been slower to develop in the biotechnology and device sectors, partly because the cost of devices is often submerged in payments to hospitals, partly because of the stunning clinical benefits afforded by some of these technologies, and partly because of the lack of alternative therapies and generics. Manufacturers should expect greater payer scrutiny of the prices for their products, however, as the technologies diffuse to the wider population and as reports surface about their actual cost. Such reports are now forthcoming from the organized buyers of these products (e.g., group purchasing organizations).

\section{Technological convergence across sectors}

Finally, this volume has been prepared in the belief that each sector must have a greater understanding of the others. The chapters have illustrated several areas in which the technologies developed by the different sectors are penetrating and, in the biological spirit of this book, "recombining" with one another to collectively add value to the healthcare provider and consumer. This has been most evident from chapters 2 and 3, which show how pharmaceutical and biotechnology firms have become increasingly interdependent in solving the twin challenges of innovation and adoption. These chapters, along with chapters $5-7$, also illustrate how drugs and devices are being combined in new treatments; how devices can be used to transport biologicals to local targeted areas in the human body; how imaging technologies can help researchers correlate biological changes with disease, provide hard endpoints to diseases, and guide implantation of surgical devices; and how broadband connectivity can communicate patient diagnostics to remote providers.

As noted in the introductory chapter, there are several barriers to growing convergence, and some of them are alluded to above. First, producers may 
eschew products with convergent technologies if they entail smaller markets than those they currently target. For such products, the innovation comes at the expense of widespread adoption. Second, convergent products may require the harmonization of different business models, cultures, and customer orientations among firms contributing to these products. As we have argued above, such harmonization is already a challenge in M\&As and strategic alliances that has not yet been successfully mastered. Third, convergence may require firms to develop a wider value chain perspective, which is not common in the healthcare industry in the US. ${ }^{10}$ This volume seeks to address this myopia.

We should point out that we have been speaking of convergence in technologies, not necessarily convergence of markets or sectors. Some discussion of the latter is in order, however. Almost all biotechnology firms (with the exception of Amgen and Genentech) and platform technology firms (with the exception of Millennium Pharmaceuticals), as cited in chapter 4, are quite some distance from developing into FIPCO-model pharmaceutical firms, but there is movement in that direction. By contrast, medical device firms are not likely to become pharmaceutical firms, even if the success of drug-eluting stents diffuses to other drug-device combination products.

To the degree that sectors actually converge, one might hypothesize what competitive dynamics might occur. The history of the convergence between the telecommunications and computer industries provides some evidence. ${ }^{11}$ Analysts anticipated that sector boundaries would become vague and blurred, the core competencies of traditional suppliers would be challenged, and firms from adjacent markets would be enticed to enter the industry as firms accumulated competencies in those markets. In fact, the convergence did not occur as expected for many of the reasons discussed in this volume. First, the presumed economies of scope from joint production were lower than expected, while the scale economies and large size of incumbents remained important advantages. Second, as discussed in chapter 5, it is difficult to make cross-firm therapeutic alliances work (let alone cross-industry market entry) when the acquiring firm does not have an historical track record in the therapeutic area and "absorptive capacity" - that is, the ability to absorb and leverage the acquired knowledge into its own technical core. ${ }^{12}$ Third, large firms are subject to inertia that reduces their ability to deal with changes in their technological cores. Indeed, the work of evolutionary theorists suggests that firm behavior is more predictable and routine than innovative, leading to the well-known "success breeds failure" syndrome. Thus, technological convergence at the market level did not play out at the firm level. 
There is another reason why convergence among sectors may not occur. The production of useful knowledge has become so specialized and professionalized that firms (and perhaps sectors too) have limited abilities to grasp and absorb it all. The range of relevant disciplines to a firm's innovative processes has expanded both in terms of the breadth of disciplines and the depth of knowledge within each one. ${ }^{13}$ Thus, the knowledge boundaries for the emerging convergent technology innovations identified above stretch way beyond a firm's production boundaries. Firms using multiple technologies to make products need to have knowledge in excess of what they need for what they produce. This imbalance is required to cope with imbalances caused by uneven rates of development in the technologies (pharmaceuticals versus biotechnology) on which they rely and with unpredictable product-level interdependencies. ${ }^{14}$ Moreover, the knowledge and product domains evolve in different ways. ${ }^{15}$ For these reasons, firms draw their organizational boundaries more tightly than they do their knowledge boundaries. ${ }^{16}$ To manage the discrepancy, they rely on the many types of strategic alliances discussed in this volume. Alliances allow them to benefit from the advantages of both specialization (scale economies) and integration (coordination). This is the more likely scenario for the future in the producer side of the healthcare industry.

\section{NOTES}

1. Robert DeGraaff, unpublished dissertation, Wharton School, 2005.

2. Robert M. Grant, Contemporary Strategy Analysis (Oxford: Blackwell, 2002).

3. Rita Gunther McGrath and Ian MacMillan, The Entrepreneurial Mindset (Boston, MA: Harvard Business School Press, 2000).

4. Andrew S. Grove, Only the Paranoid Survive (New York: Doubleday, 1996).

5. Rosabeth Moss Kanter, "Collaborative Advantage: The Art of Alliances," Harvard Business Review 72(4) (July/August, 1994): 96-107.

6. Andrew C. Inkpen, "Strategic Alliances," in Handbook of Strategic Management, ed. Michael Hitt, R. Edward Freeman, and Jeffrey S. Harrison (Oxford: Blackwell, 2001), 409-432.

7. Mitchell Lee Marks and Philip H. Mirvis, Joining Forces (San Francisco, CA: Jossey-Bass, 1998).

8. Michael Beer and Nitin Nohria, eds., Breaking the Code of Change (Boston, MA: Harvard Business School Press, 1998).

9. Cf. Stefan H. Thomke, Experimentation Matters (Boston, MA: Harvard Business School Press, 2003).

10. Lawton R. Burns, The Health Care Value Chain (San Francisco, CA: Jossey-Bass, 2002). 
11. Geert Duysters and John Hagedoorn, "Technological Convergence in the IT Industry: The Role of Strategic Technology Alliances and Technological Competencies," unpublished manuscript, Maastricht University, 1997.

12. W. M. Cohen and Daniel Levinthal, "Absorptive Capacity: A New Perspective on Learning and Innovation," Administrative Science Quarterly 35 (1990): 128-152.

13. Q. Wang and G. N. Von Tunzelmann, "Complexity and the Functions of the Firm: Breadth and Depth," Research Policy 29 (2000): 805-818.

14. Stefano Brusoni, Andreas Prencipe, and Keith Pavitt, "Knowledge Specialization, Organization Coupling, and the Boundaries of the Firm: Why do Firms Know More than they Make?," Administrative Science Quarterly 46 (December, 2001): 597-621.

15. G. N. Von Tunzelmann, "Localised Technological Search and Multitechnology Companies," Economics of Innovation and New Technology 6 (1998): 231-255.

16. Brusoni et al. "Knowledge Specialization." 